\title{
Bilan de $\mathbf{3 0}$ ans de travaux de recherche effectués en France sur la pollinisation du colza
}

\author{
Jaqueline PIERRE ${ }^{1}$ \\ Michel RENARD ${ }^{2}$ \\ ${ }^{1}$ INRA UMR 1099 BiO3P \\ (Biologie des Populations appliquée \\ à la Protection des Plantes), \\ Domaine de la Motte, BP 35327, \\ F 35653 Le Rheu \\ $<$ jacqueline.pierre@rennes.inra.fr> \\ ${ }^{2}$ UMR 118, \\ APBV (Amélioration des Plantes \\ et Biotechnologies Végétale), \\ INRA Domaine de la Motte, BP 35327, \\ F 35653 Le Rheu France \\ <michel.renard@rennes.inra.fr>
}

Les dernières informations du GEVES ${ }^{1}$ confirment la création de 100 nouvelles variétés de colza depuis trois ans. II s'agit à la fois de variétés classiques mais aussi et surtout actuellement de variétés hybrides. Les débouchés du colza se sont au fil des années affinés et diversifiés : utilisation de tourteau plus appétant pour l'alimentation animale, utilisation d'huiles sans acide érucique riches en oméga- 3 et - 6 pour I'alimentation humaine, utilisation d'huiles de qualité spécifique par l'industrie, enfin utilisation agro-environnementale du colza comme pompe à nitrate. En France, cette diversification est le fruit de recherches d'amont conduites par l'INRA en collaboration avec des partenaires du privé dont le CETIOM est le principal représentant.

Cet article rapporte le bilan de près de 30 ans de travaux effectués à l'INRA de Rennes sur la pollinisation du colza (1980-2010). En effet, la sélection de qualités intrinsèques chez les lignées (taille de la plante, morphologie florale, teneur en huile, teneur en glucosinolates et acide érucique, résistance aux maladies, résistance aux herbicides) qui déterminent et favorisent les débouchés du colza, s'est accompagnée d'une évolution des modalités de polli-

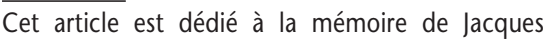
Mesquida.

${ }^{1}$ Groupe d'Etude des Variétés et des Semences

\begin{abstract}
This article is a report of nearly 30 years of investigations made at INRA Rennes on oilseed rape pollination. During that years, the cultivars diversified and with them the pollination needs. Two apparently contradictory purposes were followed: i) to optimize the pollen transfer to produce hybrid seed, ii) to reduce pollen transfer to warrant pure seeds production or to limit GMO pollen dispersal. Knowing that pollen transfers are dur to insects and wind, the role of the two vectors was respectively studied. Briefly here we show that the role of insects has often been underestimated even in the pollination of conventional (hermaphroditic) oilseed rape and that the pollen of oilseed rape is not suited to wind dispersal and is therefore transported at only short distance.
\end{abstract}

Key words: oilseed rape, pollination, honeybee, wind, seed production nisation (pollinisation classique versus hybridation). Il est donc apparu nécessaire d'étudier les conséquences positives ou négatives de ces innovations pour gérer les modalités de pollinisation afin de garantir qualitativement et quantitativement la production.

\section{Des colzas classiques aux colzas hybrides}

Le colza classique est une plante hermaphrodite dont le mode de reproduction est considéré comme partiellement autogame car la plante est protogyne, ce qui implique que le stigmate d'une fleur est réceptif avant ses propres anthères et par conséquent pollinisable par les fleurs plus âgées de la même plante ou celles de plantes voisines. Le taux d'allogamie moyen habituellement cité est de $30 \%$ mais la littérature indique des taux d'allogamie extrêmement variables (de 0 \% à 90 \%) selon les variétés, le dispositif expérimental, les conditions environnementales et le caractère marqueur utilisé car ce dernier est repéré avec plus ou moins de fiabilité dans les croisements (Downey et Röbbelen, 1989 ; Rakow et Woods, 1987 ; Lewis et Woods, 1991). Néanmoins, compte tenu de la manière dont les semences sont produites, à savoir sur des surfaces homogènes suffisamment grandes et avec des distances d'isolement imposées par la régle- mentation, on peut considérer que l'autogamie au sens large (croisement entre plantes d'une même variété) est la règle générale ce qui conduit à une pureté satisfaisante des variétés (Klein et al., 2006).

À l'opposée, I'allogamie a été favorisée comme mode de reproduction depuis que l'effet d'hétérosis a été mis en évidence chez le colza (Lefort-Buson et al., 1987). La création de lignées femelles est alors apparue comme un stade décisif pour produire des semences hybrides. Plusieurs systèmes conduisant à la stérilité mâle du colza ont été exploités à cette fin dans divers pays mais nous n'évoquerons ici que la stérilité mâle génocytoplasmique mise au point par I'INRA de Rennes et de Versailles dans les années 1980. Dès lors la question s'est posée du transfert de pollen entre les plantes mâles (hermaphrodites) et les plantes femelles ou mâle-stériles, à savoir un transfert sur des distances moyennes dépassant le simple cadre de la plante ou de ses voisines immédiates.

\section{Le transfert du pollen des plantes mâles vers les plantes femelles}

Cette question a été abordée selon deux volets complémentaires: i) quels sont les vecteurs de pollen?, et ii) comment mettre en place un dispositif permettant à ces vecteurs de 
jouer au mieux leur rôle tout en sachant que les semences hybrides ne sont récoltées que sur les plantes femelles?

Concernant les vecteurs de pollen, il était traditionnellement admis que l'allo-pollinisation du colza était effectuée à la fois par le vent et par les insectes, sans que la part respective de chacun ne soit d'ailleurs à l'époque, vraiment évaluée. Néanmoins, il semblait évident qu'il fallait prendre en compte le comportement des abeilles vis-à-vis des fleurs mâle-stériles. Ceci a été l'objet des travaux de Mesquida et Renard dès les années 1980. En effet les insectes pollinisateurs tels que les abeilles ou les bourdons butinent la fleur pour y collecter du pollen et du nectar. Or les fleurs mâle-stériles ne fournissent pas de pollen et sont donc supposées être moins attractives. De plus, I'utilisation de la stérilité mâle génocytoplasmique OGU-INRA s'est accompagnée, dans un premier temps, d'un déficit en nectar entraînant une polarisation des butineuses sur les bandes mâles (Renard et Mesquida, 1983). Cette déficience a ensuite été corrigée en créant des cybrides par fusion de protoplastes (Pelletier et al., 1983) qui globalement ont produit $70 \%$ de nectar par rapport au témoin mâle-fertile sans modification de la teneur et de la composition en sucres (Mesquida et al., 1991). Un bilan effectué ultérieurement sur 71 génotypes différents (mâle-fertiles; simple zéro, double zéro, mâlestériles) a montré que les lignées double zéro sécrétaient davantage de nectar que les autres et a confirmé que les lignées femelles tendaient à produire moins de nectar (Pierre et al., 1999). Cependant, I'amélioration des secrétions chez les plantes femelles cybrides a induit une meilleure répartition des abeilles entre bandes mâles et bandes femelles (semées en alternance) ce qui s'est traduit par un rendement en grains relativement homogène des bandes femelles jusqu'à une distance de $30 \mathrm{~m}$ par rapport à la bande mâle (Renard et Mesquida, 1987 ; Renard et al., 1987).

Par la suite, les colzas hybrides se sont diversifiés avec la création d'hybrides restaurés (Delourme et al., 1995) ou bien encore d'associations variétales comprenant les composites hybrides lignées et les hybrides mixtes (Pierre et Renard, 1995). Fondamentalement, du point de vue de la pollinisation et de la production de semences hybrides, le problème est resté le même: fournir aux abeilles des plantes femelles suffisamment riches en nectar pour qu'elles assurent leur rôle de vecteur de pollen en passant de manière fréquente entre les plantes mâles et femelles. Sous ce rapport il a été montré que les lignées restauratrices offraient autant de nectar que les colza mâlefertiles classiques (Pierre et al., 1999).

Tout récemment les apiculteurs bretons se sont émus des faibles miellées enregistrées sur colza.
Si tel était le cas, au-delà des conséquences que cela pourrait avoir pour cette profession, cela pourrait impliquer une baisse de rendement en production de semences hybrides du fait de la désertion du champ par les abeilles. Compte tenu de nos travaux antérieurs, nous avons fait l'hypothèse que certains génotypes actuels pourraient avoir une faible production en nectar, ce critère n'étant pas pris en compte dans la sélection. Après vérification de la production nectarifère des variétés classiques ou des lignées parentales des associations variétales incriminées, il est apparu que la production de nectar était satisfaisante et que les conditions climatiques expliquaient principalement les déficits d'abeilles enregistrés (Pierre et Emeillat, 2009). De plus, cette étude a permis de mettre en exergue le fait que même si la production de nectar par fleur est plus faible chez les plantes femelles la densité de fleurs est telle que la quantité de nectar fournie par $\mathrm{m}^{2}$ est considérable et largement du niveau de bien d'autres plantes considérées comme mellifères.

\section{La pollinisation de lignées colzas ayant une morphologie particulière}

Parmi les types de développement particulier on peut citer les colzas nains ou les colzas dont la morphologie florale a été modifiée (colza sans pétales, colza à fleurs fermées).

L'observation du comportement des abeilles sur les colzas nains a surtout montré que les abeilles évitaient de passer d'un colza nain vers un colza de taille plus grande (différence supérieure à $50 \mathrm{~cm}$ ) quand ceux-ci se trouvaient côte à côte. Ceci est d'autant plus marqué si à la différence de taille est associée une différence de production de nectar. Ainsi, les passages se trouvent en moyenne réduits de 40 à $20 \%$ entre un mâle fertile normal et une femelle naine ou un mâle nain et une femelle de taille normale. Malgré cela, il est possible de produire avec de bons rendements des semences d'hybrides demi-nains dans des dispositifs en bandes alternées, comprenant soit un mâle nain soit une femelle naine (Pierre et al., 1999).

La modification de la morphologie florale s'est révélée par contre avoir une incidence beaucoup plus nette sur le comportement des abeilles. Les lignées de colza sans pétales, créées dans le but d'éviter les attaques de sclérotiniose (Lamarque, 1983) ou d'augmenter le rendement (Mendham et al., 1991) se sont révélées être tout à fait attractives pour les abeilles. Malgré leur absence de pétales, elles sont visitées tout autant que des lignées classiques car elles produisent des quantités normales de nectar et de pollen. Cependant, une observation plus fine des comportements a montré que les abeilles adoptaient une posture particulière pour prélever le nectar sur ce type de fleurs; à savoir un butinage latérale sans contact avec les étamines entraînant une forte réduction de la charge en pollen sur le corps de l'abeille et par la suite un faible transfert (Pierre et Renard, 1994; Pierre et Renard, 1997). De manière encore plus significative pour la pollinisation, il est apparu que les abeilles se spécialisaient individuellement pour le butinage d'un type de fleur. Ainsi, les passages d'abeilles d'une fleur apétale vers une fleur avec pétales et inversement sont considérablement réduits voire quasi nuls (Pierre et al., 1996). Cette forte fidélité de l'abeille à un type floral, y compris au sein d'une même espèce végétale, peut être exploitée pour préserver la pureté variétale notamment pour la production de semences de colzas ayant des qualités spécifiques.

Cette spécialisation du butinage et la fidélité à une morphologie florale a également été constatée pour les fleurs fermées (cléistogames): le butinage du nectar de ces fleurs est effectué latéralement par les interstices présents à la base de la corolle. Dans ce cas, les abeilles ne cherchent pas à ouvrir la fleur pour obtenir du pollen qu'elles vont par ailleurs chercher sur $\mathrm{d}^{\prime}$ autres fleurs. Elles n'ont de ce fait aucun contact avec les étamines de sorte qu'elles ne constituent pas un péril pour l'utilisation de telles lignées (Pierre et Renard, 1999). En effet, des lignées cléistogames ont été créées par mutation induite à I'INRA de Rennes (Renard et Tanguy, 1997 ; Brevet FR 07.08049, 2007) à la fois pour réduire la dispersion de pollen et favoriser l'autofécondation.

\section{Le cas des colzas OGM}

La création de colzas cléistogames a été motivée par le souci de favoriser la coexistence entre colzas conventionnels et colzas génétiquement modifiés ou de qualité spécifique. En effet, dans les années 1990, diverses lignées expérimentales de colza OGM ont été testées puis des colzas tolérants aux herbicides sont apparus sur le marché. Durant cette période, les expérimentations ont porté à la fois sur les risques que ces colzas pourraient présenter pour la santé de l'abeille mais aussi sur les risques de croisements intraspécifiques et interspécifiques entre plantes génétiquement modifiées et plantes classiques. Corrélativement, cela a conduit à reconsidérer le rôle des abeilles dans les transferts de pollen, l'objectif étant cette fois-ci de réduire leur rôle.

L'étude de la toxicité de transgènes (introduits dans le colza ou autre) vis-à-vis des abeilles a fait l'objet de réflexions méthodologiques 
(Pierre et Pham-Delègue, 2000). De nombreux travaux de recherche portant, durant plusieurs années, soit sur des OGM existants ou des OGM expérimentaux ont fait l'objet de synthèses (Couty et al., 2005). Une revue bibliographique des travaux effectués principalement à I'INRA de Bures-sur-Yvette et de Rennes ou dans des laboratoires étrangers montre que les transgènes utilisés à des fins insecticides n'ont pas d'incidence notable sur la santé des abeilles (Pierre, 2007). D'autres études ont également porté sur des colzas tolérants à divers herbicides. Bien que les transgènes utilisés n'aient normalement aucun lien avec les insectes, les études ont été faites dans le souci de vérifier si des effets indésirables ne risquaient pas d'apparaître par effet de pléiotropie (effet sur la production de nectar ou de pollen par exemple). Les résultats portant sur la résistance au glufosinate (transgène pat) ont en particulier montré que la production de nectar et de pollen ainsi que le comportement des butineuses n'étaient en rien modifiés par le transgène et que celui-ci n'avait pas d'incidence sur l'abondance et la diversité de l'entomofaune pollinisatrice (Marsault et al., 2001 ; Pierre et al., 2003).

Pour aussi rassurants que soient de tels résultats, ils conduisent à d'autres questions: si les abeilles et autres pollinisateurs ne font aucune différence entre colza OGM et non OGM quelles sont les conséquences de leur capacité à véhiculer du pollen OGM comme s'il s'agissait d'un non OGM? Quels sont les risques de croisements et les impacts agroenvironnementaux? (Chèvre et al., 1998; Chèvre et al., 2001). Ainsi, parallèlement aux travaux sur la toxicité, des travaux sur la dispersion du pollen ont été entrepris. Qu'il s'agisse de recherches de I'INRA ou bien de recherches conduites par d'autres nombreuses équipes internationales, les études de la dispersion de pollen dans des dispositifs continus, suivie par marqueur, montrent une rapide décroissance de la contamination en fonction de la distance par rapport à la source contaminante avec des taux de contamination très faibles à $70 \mathrm{~m}$ (Lavigne et al., 1998). Audelà de cette distance, des pollutions existent qui semblent aléatoires malgré les tentatives d'explication proposées par certains auteurs.

Devant une telle difficulté à comprendre et contrôler la dispersion du pollen, les recherches se sont orientées vers la modélisation de la dispersion et diverses expérimentations selon des dispositifs discontinus faisant appel soit à des zones nues, soit à des distances d'isolement, soit à des bordures de plantes dites " pièges » (pour une revue voir Damgaard et Kjellson, 2005). Dans de telles expériences aucune distinction n'a été établie entre la vection du pollen par les insectes ou par le vent.
Par contre, les travaux conduits à l'INRA se sont focalisés sur l'étude du déplacement individuel des abeilles et de leur comportement face à une zone vide, en partant du principe que chaque individu véhicule du pollen. Le suivi individuel d'abeilles marquées a montré que leur aire de butinage était très limitée (environ $100 \mathrm{~m}^{2}$ ) et que chacune restait fidèle à son aire de butinage entre deux déplacements De plus, il est apparu que les abeilles évitaient de traverser une zone vide entre deux bandes de colza de même morphologie (Pierre et Renard, 1999). Ces résultats plaidaient en faveur de l'utilisation de zones nues pour éviter les transferts de pollen tandis que d'autres résultats montraient qu'au contraire une zone nue pouvait favoriser les flux de pollen sans démontrer si la zone nue avait un effet sur le vent ou les insectes (Morris et al., 1994). Nos recherches se sont alors portées sur l'existence d'échanges de pollen entre abeilles au sein de la ruche (Dag et al., 2001). Dans ce cas, l'hypothèse est faite qu'une abeille butinant un colza A sur un lieu $A$ et une abeille butinant un colza $B$ sur un lieu B peuvent échanger accidentellement leur pollen dans la ruche. Un dispositif sous cage pollen-proof avec des ruches à double entrée connectée d'une part à la cage semée de plantes mâle-stériles et d'autre part vers l'extérieur sur un champ de colza mâle fertile a montré que de tels échanges pouvaient avoir lieu et permettre la pollinisation des plantes mâle-stériles dans la cage. La difficulté de ce type de résultats est qu'il montre que le phénomène existe mais il est impossible d'en déduire une probabilité du risque de contamination dans les conditions de culture.

Concernant les risques de croisements interspécifiques, la démonstration par diverses équipes que des croisements étaient possibles entre le colza et certains apparentés en particulier la ravenelle (Chèvre et al., 1997) nous a incité à étudier le comportement des insectes pollinisateurs sur cette dernière espèce (Raphanus raphanistrum). Les résultats montrent que la ravenelle est visitée par les abeilles car elle fournit pollen et nectar en quantité normale. Par contre, quand les abeilles ont la possibilité de choisir entre colza et ravenelle, le colza est préféré à la ravenelle car la corole de celle-ci, plus étroite et plus profonde, rend son butinage plus difficile. Dans une situation où I'on place $1 \mathrm{~m}^{2}$ de ravenelle au centre d'un champ de colza de $100 \mathrm{~m} \times 100 \mathrm{~m}$, les abeilles évitent systématiquement la ravenelle pour des raisons de fidélité à la fleur déjà décrite plus haut. En revanche, les insectes tels que les mouches ou les espèces de bourdons à langue longue sont beaucoup moins sélectifs. Dans la mesure où ils ne représentent qu'un faible pourcentage de la population d'insectes lorsque des ruches sont apportées (moins de $5 \%$ ), leur rôle dans les transferts interspécifiques de pollen est cependant réduit (Pierre, 2001).

\section{Quelle est la part respective de pollinisation due au vent et aux insectes ?}

Parmi les travaux cités ci-dessus, peu ont permis de dissocier la part de pollinisation due au vent ou aux insectes. Ceci n'est possible qu'en effectuant des expérimentations comparatives en mettant ou non les plantes sous tulle ou sous cage de manière à exclure ou non les insectes de la pollinisation. Par des expérimentations sous cage, Mesquida et al. (1988) ont montré que les abeilles jouaient un rôle majeur dans la pollinisation. La limite de telles expérimentations résulte de l'effet cage sur le vent. Des travaux beaucoup plus récents, effectués au champ, ont consisté à empêcher la visite des fleurs par les insectes en mettant uniquement quelques inflorescences sous tulle de manière à perturber le moins possible la physiologie de la plante. Là encore, le rôle des abeilles se révèle considérable y compris dans la pollinisation des fleurs hermaphrodites. Leur contribution à la production de graines dans ce cas est de $36 \%$ et atteint $90 \%$ pour la production de semences hybrides (Pierre et al., 2010). Ces résultats confirment des travaux antérieurs ou ceux d'autres auteurs qui considèrent que le vent est un mauvais vecteur du pollen de colza (Eisikowitch, 1981 ; Mesquida et Renard, 1982; Cresswell et al., 2003). En fait, le pollen frais de colza est collant et tend à rester en agrégats. Nous avons montré récemment que les abeilles participent à la dissémination aérodynamique de ce pollen par largage et provoquent la pollinisation de fleurs femelles placées sous tulle sans avoir le moindre contact avec la fleur à polliniser (Pierre et al., 2010). C'est la première fois que l'existence d'une pollinisation anémophile assistée par les insectes est démontrée.

\section{Notion de pollen efficace}

L'idée selon laquelle le pollen de colza est anémophile a été largement entretenue par le fait que l'on retrouve du pollen de colza sur des filtres pièges à de grandes distances de la source. En réalité, collecter du pollen par piégeage ne donne aucune indication quant à son âge et son efficacité pollinisatrice. II faut tenir compte de la durée de vie du pollen dans différentes conditions si l'on veut être en mesure d'apprécier son pouvoir fécondant dans diverses situations. Bien que les mesures de viabilité du pollen soient longues et difficiles 
à réaliser, Mesquida et al. (1987) ont trouvé une perte de l'aptitude à germer in vitro au bout de 3 jours; en utilisant une autre technique Pierre et Renard, 2002 ont trouvé une longévité d'environ 7 jours in vivo sachant par ailleurs que cette longévité dépendait des conditions climatiques. Actuellement une amélioration des techniques de mesure de viabilité est en cours (Hmimina et al., en prép.) et les résultats confirment une longévité (in vitro) de l'ordre de 9 jours à température ambiante et de 18 jours à $4{ }^{\circ} \mathrm{C}$, ceci à des hygrométries inférieures à $20 \%$. Cependant, la question reste posée de savoir quelle est la longévité des grains de pollen qui parcourent de longue distance après désagrégation et ascendance dans les couches supérieures de l'atmosphère, puis leur retombée.

La longévité du pollen sur le corps de l'abeille a également fait l'objet d'études (Mesquida et Renard, 1989). Ces travaux ont montré une baisse importante du pouvoir germinatif après passage sur le corps de l'insecte (perte de 60 à $85 \% 2$ heures après le butinage). À noter qu'il s'agit du pollen «libre » accroché dans la toison et non pas du pollen des pelotes qui, étant totalement aggloméré, n'est pas disponible pour la pollinisation. Cette connaissance de la brièveté de la durée de vie du pollen sur le corps de l'insecte laisse supposer que la pollinisation entomophile n'est réalisée que si l'abeille transfère rapidement le pollen entre les plantes. On sait également qu'une centaine de grain suffisent pour féconder la vingtaine d'ovules du colza (Mesquida et Renard, 1984) ceci ayant été confirmé dans le cas de la production de semences hybrides chez le chou-fleur. Cette quantité correspond, par exemple, à ce qui est déposé lors d'une ou deux visites par des bourdons.

\section{Faut-il apporter des ruches ou non pour favoriser la pollinisation?}

La prise en compte de la participation relative du vent et des insectes à la pollinisation est différente selon que l'on se préoccupe de favoriser la pollinisation pour la production de semences ou au contraire que l'on souhaite éviter la dissémination du pollen.

Par rapport à la production de semences, l'apport de ruche est un élément favorisant la synchronisation de la fructification (Mesquida et Renard, 1981) et, par la suite, la qualité des semences. II est difficile de comprendre les phénomènes de compensation entre les diverses composantes du rendement (Mesquida et Renard, 1981) mais il semble logique de considérer que lorsque les conditions climatiques sont favorables celles-ci le sont à la fois pour la plante et l'activité des abeilles et que par conséquent la fécondation se fait dans des conditions optimales. Cet impact positif de l'action des abeilles est évident pour la production de semences hybrides sous réserve que l'alternance de bande mâles et femelles soit appropriée. En effet, le rôle du vent est limité à quelques mètres et même si les abeilles participent à plus de $90 \%$ à la pollinisation des bandes femelles, la production de grains diminue rapidement avec la distance car les abeilles qui se sont chargées en pollen sur les bandes mâles perdent progressivement leur charge en pollen car elles visitent les bandes femelles suivantes sans revenir sur

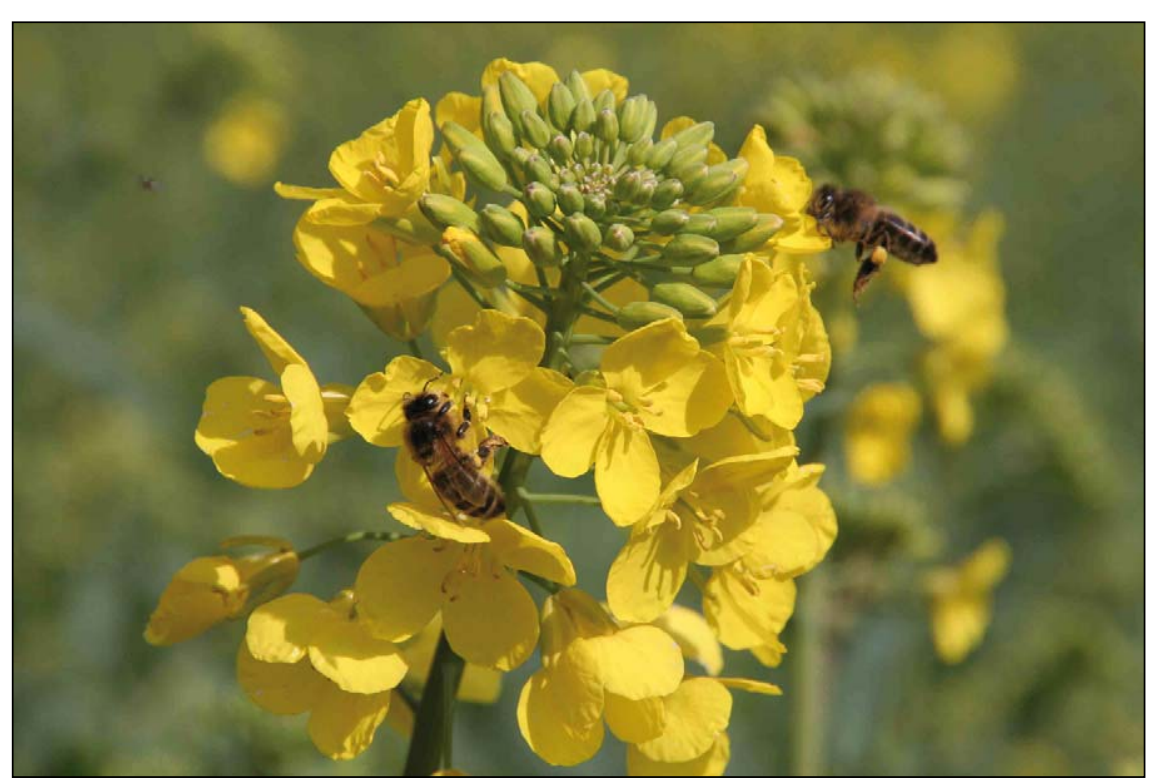

Figure 1. Abeilles domestiques butinant une fleur de colza. Photo INRA.

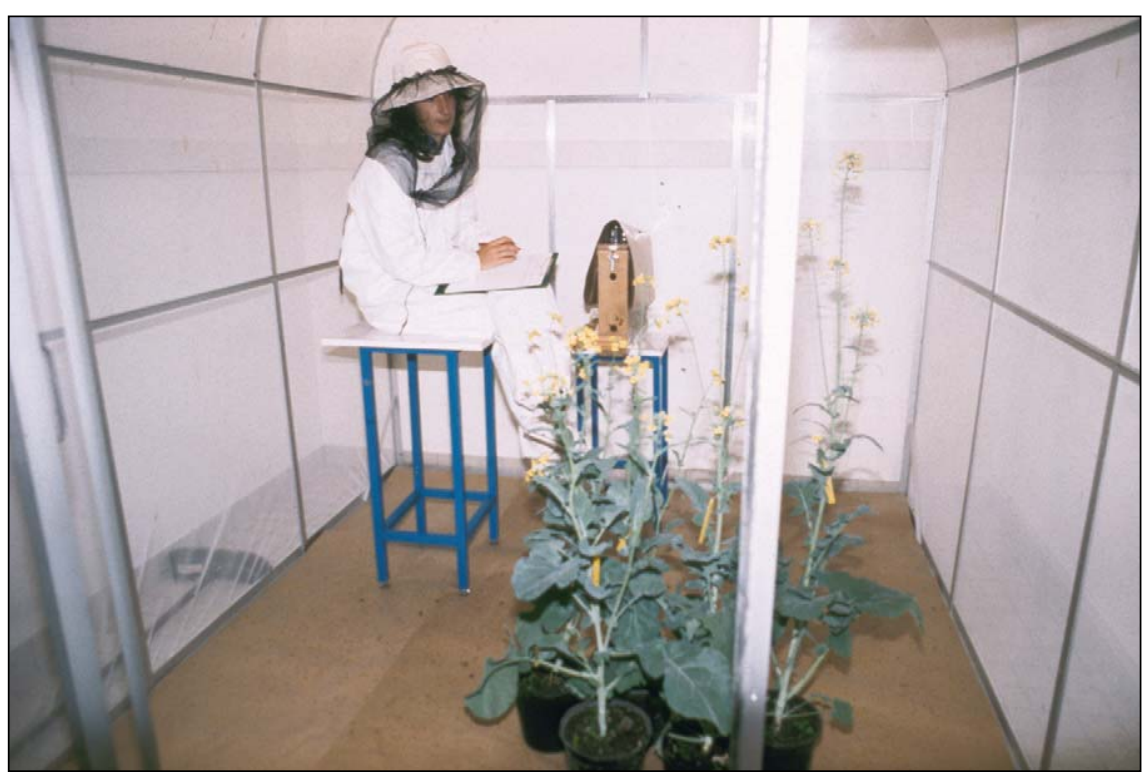

Figure 2. Étude au laboratoire du comportement de butinage des abeilles sur des plants de colza. Photo INRA. 


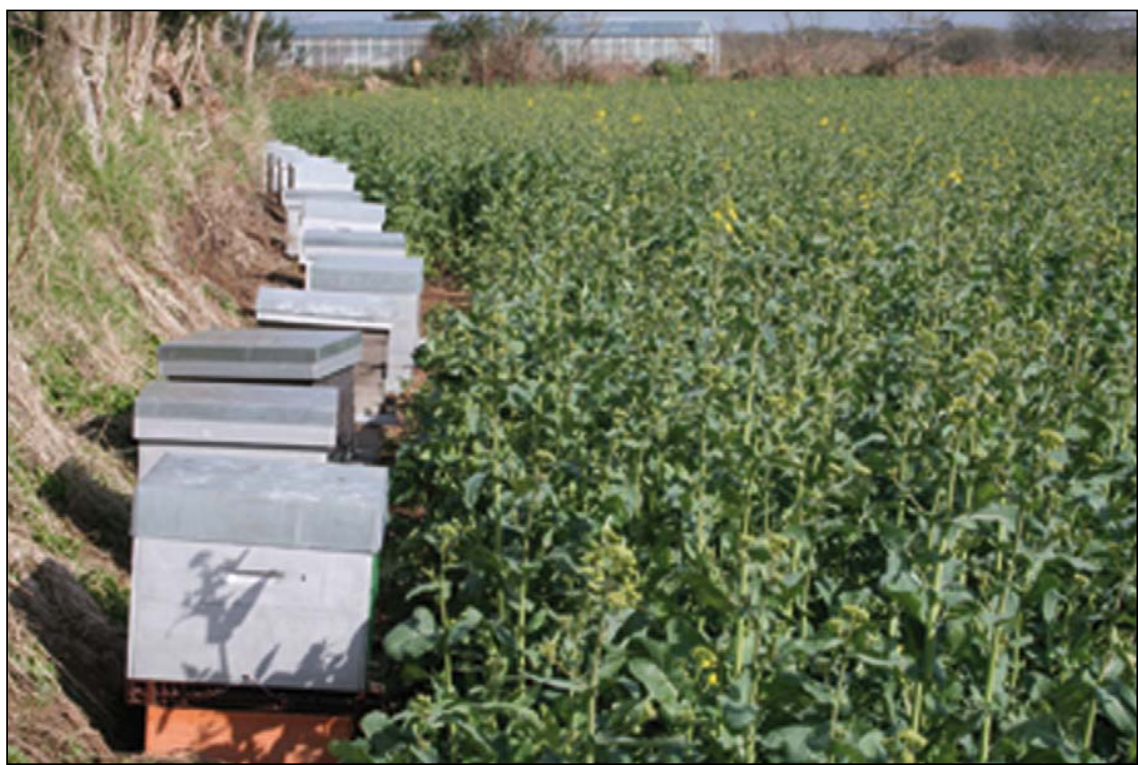

Figure 3. Ruches dans un champ de colza. Photo INRA.

Pham-Delègue, 2009). Mettre en avant l'impact sur la stabilité du rendement en situations de contraintes environnementales limitant les effets de compétition?

Par contre, par rapport aux risques de dissémination de pollen indésirable, on peut estimer $a$ priori que ne pas apporter de ruches permettrait de réduire ces risques. Sans vouloir faire un plaidoyer quelconque pour les abeilles domestiques, il est tout à fait défendable de proposer l'hypothèse inverse : I'apport massif $d^{\prime}$ abeilles qui sont fidèles à un type de fleur et à leur site de butinage et qui entrent en compétition avec d'autres pollinisateurs au comportement plus erratique (mouches ou abeilles solitaires) offrirait plutôt une garantie de diminution des contaminations. La pollinisation avec abeilles se faisant dans des délais plus rapides et sans prolongement de la floraison, les risques de dispersion de pollen dans le temps seraient moindres, sous réserve de conditions climatiques favorables. Reste une incertitude sur les risques liés aux échanges de pollen intraruches mais ceux-ci devraient rester faibles sachant que le pollen mis en contact avec les abeilles voit sa longévité raccourcie. Reste aussi une incertitude quant au rôle joué par ces quelques abeilles particulières que sont les éclaireuses et qui ont pour fonction d'explorer l'environnement pour y trouver des ressources et dont le comportement de butinage est mal connu. Enfin, on peut considérer que les butineuses classiques ne présentent pas un danger pour l'utilisation des lignées cléistogames, puisqu'il a été montré que les abeilles n'ouvrent pas les fleurs fermées de ces lignées créées afin d'éviter la dispersion de pollen. Même en cas d'instabilité de la fermeture ce système, I'allogamie se trouve réduite de $95 \%$ (Pierre et al., 2010), ce qui conduit à un taux de contamination final très satisfaisant par rapport à la réglementation européenne.

\section{Encore des interrogations}

L'originalité des travaux réalisés par nos deux équipes résulte principalement de l'originalité même du matériel végétal étudié et de nos compétences complémentaires en génétique et en comportement des insectes, mais également de la contribution de partenaires couvrant des domaines très divers allant de la modélisation à la production de semences.

Cette collaboration continue depuis près de 30 ans a permis d'acquérir des connaissances sur la pollinisation du colza et de proposer entre autres des procédures efficaces de production de semences hybrides de colza. II reste cependant encore bien des interrogations quant à la pollinisation du colza, en particulier en ce qui concerne les taux variables d'allogamie enregistrés par les différents auteurs chez les variétés de colza classique. De même, les travaux sur la physiologie de la reproduction restent encore insuffisants : on ignore par exemple quelles sont les conditions environnementales qui préparent à une bonne fertilité du pollen et des ovules, s'il y a des colzas plus ou moins protogynes. II reste aussi des points $d^{\prime}$ 'incertitude relatifs au risque de flux de gènes à distance. Ces flux se font a priori essentiellement par les graines (Colbach et al., 2001), et nous avons vu que pour ce qui est de la dissémination par le pollen des solutions très prometteuses existent dont I'utilisation de la
(Fargue et al., 2006 ; Leflon et al., 2009), par cléistogamie, laquelle fait maintenant l'objet d'études menées par différentes équipes en Europe.

\section{RÉFÉRENCES}

Chèvre AM, Eber F, Baranger A, Vallée P, Pierre I, Renard M. Impact de la transformation génétique du colza : Synthèse. Cahiers de l'Agriculture 1998 ; 7 : 525-30.

Chèvre AM, Eber F, Baranger A, Renard M. Gene flow from transgenic crops. Nature 1997 ; 389 : 924.

Chèvre AM, Eber $F$, Jenczewski $E$, et al. Impact agroenvironnementaux de la mise en culture de variétés de colza transgéniques tolérantes à des herbicides. CR Académie d'Agriculture Française 2001; 87 : 11-20.

Colbach N, Clermont-Dauphin C, Meynard JM. GeneSys: a model of the influence of cropping system on gene escape from herbicide tolerant rapeseed crops to rape volunteers II. Genetic exchanges among volunteer and cropped populations in a small region. Agric Ecosyst Environ $2001 ; 83$ : 255-70.

Couty A, Pierre I, Pham-Delègue MH. Effets non intentionnels des plantes transgéniques sur les insectes non cibles. In : Regnault-Roger C, ed. Enjeux phytosanitaires pour l'agriculture et l'environnement. France : Lavoisier, 2005.

Cresswell, JE, Davies TW, Patrick MA, Russell F, Pennel C, Vicot M, Lahoubi M. Brassica napus is aerodynamically unsuited to cross pollination by wind. In : Proc. of the $1^{\text {st }}$ conference on the Coexistence of Genetically Modified Crops with conventional and organic crops. 13-14 November 2003, Ed. Boelt.

Dag A, Degani C, Gazi S. In hive pollen transfer in Mango. In: Proc. $8^{\text {th }}$ Pollination Symp. Benedek \& Richards (eds). Acta Hort 2001; $561: 61$.

Damgaard C, Kjellson G. Gene flow of oilseed rape (Brassica napus) according to isolation distance and buffer zone. Agric Ecosyst Environ 2005; 108 : 291-305.

Delourme R, Eber F, Renard M. Breeding double low restorer lines in radish cytoplamic male sterility. In : Proc IXth International Rapeseed Congress. Cambridge, UK, 4-7 july 1995.

Downey RK, Röbbelen G. Brassica species. In : Röbbelen G, Downey RK, Ashri A, eds. Oil crops of the world. New-York (USA) : Mc Graw-Hill, 1989.

Eisikowitch D. Some aspects of pollination of oilseed rape (Brassica napus L.). J Agric Sci Cambridge 1981 ; $96: 321-6$.

Fargue A, Colbach N, Pierre J, Picault H, Renard M, Meynard JM. Predictive study of the advantages of cleistogamy in oilseed rape in limiting unwanted gene flow. Euphytica $2006 ; 151: 1-13$.

Hmimina G, Sourisseau A, Pierre J, Ricroch A. Assessing oilseed rape pollen viability for improving dessicated tricellular pollen storage at low temperature. (2010 en prep.). 
Klein E, Lavigne C, Piault H, Renard M, Gouyon PH. Pollen dispersal of oilseed rape : estimation of the dispersal function and effects of field dimension. J Appl Biol 2006 ; 43 : 141-51.

Lamarque $\mathrm{C}$. Conditions climatiques qui favorisent le processus naturel de contamination du colza par le sclerotinia sclerotiorum. In : Proc. International Rapeseed Congress, 1983, Paris, France.

Lavigne C, Klein E, Vallée P, Pierre J, Godelle B, Renard $M$. A pollen-dispersal experiment with transgenic oilseed rape. Estimation of the average pollen dispersal of an individual plant within a field. Theor Appl Genet 1998 ; 96 : 886-96.

Leflon M, Hüsken A, Njontie C, et al. Stability of the cleistogamous trait during the flowering period of oilseed rape. Plant breeding $2009 ; 129$ : 13-8.

Lefort-Buson M, Guillot-Lemoine B, Dattée Y. Heterosis and genetic distance in rapeseeed (Brassica napus L.). Crosses between european and asiatic lines. Genome 1987 ; 29 : 413-8.

Lewis LI, Woods DL. Outcrossing in summer oilseed rape (Brassica napus L.) under Peace River Alberta conditions. In : Proc GCIRC Congress 9-10 July 1991 Saskatoon Saskatchewan Canada

Manning R, Wallis IR. Seed yields in canola (Brassica napus $\mathrm{cv}$. Karoo) depends on the distance of plants from honeybee apiaries. Austral J Exp Agric 2005 ; 45 : 1307-13.

Marsault D, Pierre J, Pham-Delègue MH. Biodiversity and foraging behaviour of pollinating insects on herbicide-tolerant transgenic oilseed rape under field conditions. Proc International Union for the Study of Social Insects. September 25-29, 2001, Berlin, Germany.

Mendham NJ, Rao MSS, Buzza GC. The apetalous flower character as a component of a high yielding ideotype. In: Proc VIIlth International Rapeseed Congress 1991. Saskatoon Canada : 596-600.

Mesquida J, Pham-Delègue MH, Marilleau R, Le Métayer $M$, Renard M. La sécrétion nectarifère des fleurs de cybrides mâle-stériles de colza d'hiver (Brassica napus L.). Agronomie 1991 ; 11 : 217-27.

Mesquida J, Renard M. Etude de l'aptitude à germer in vitro du pollen de colza (Brassica napus L.) récolté par l'abeille domestique (Apis mellifica L.). Apidologie $1989 ; 20$ : 197-205.

Mesquida J, Renard M, Mesquida B. Etude préliminaire sur la germination in vitro du pollen de colza (Brassica napus L. var. oleifera Metzger) et sur l'évolution dans le temps de son aptitude à germer. Agronomie 1987 ; 7 : 409-16.

Mesquida J, Renard M. Pollinisation du colza d'hiver mâle-fertile et mâle-stérile (Brassica napus L. var. oleifera Metzger) par l'abeille domestique (Apis $m$. mellifica $\mathrm{L}$.). Effets sur la phénologie et le rendement. Apidologie 1981 ; 12 : 345-62.

Mesquida J, Renard M. Etude de la dispersion du pollen par le vent et de l'importance de la pollinisation anémophile chez le colza (Brassica napus L.; var oleifera Metzger). Apidologie 1982 ; 13 : 353-66.
Mesquida J, Renard M. Etude des quantités de pollen déposées sur les stigmates dans différentes conditions de pollinisation; influence sur la production de graines chez le colza d'hiver mâle-fertile. Vème Symposium International sur la Pollinisation, Versailles 27-30 Septembre 1983, INRA éditions, 1984.

Mesquida J, Renard M, Pierre JS. Rapeseed Brassica napus L. productivity: the effect of honeybees Apis mellifera $\mathrm{L}$. and different pollination conditions in cage and field tests. Apidologie $1988 ; 19: 51-72$.

Morris WF, Kareiva PM, Raymer PL. Do baren zone and pollen traps reduce gene escape from transgenic crops. Ecol Appl $1994 ; 4$ : 157-65.

Pelletier G, Primard C, Vedel F, Remy R, Roussel P. Intergeneric cytoplasmic hybridization in Cruciferae by protoplast fusion. Mol Gen Genet 1983; 191 : 244-50.

Pierre J. The role of honeybees (Apis mellifera) and other insect pollinators in gene flow between oilseed rape (Brassica napus) and wild radish (Raphanus raphanistrum). In : Proc. 8th International Pollination Symposium, Benedek P, Richards KW eds, Acta Horticulturae, 2001; 47-51.

Pierre J. Les abeilles et les colzas OGM : revue bibliographique. La santé de l'abeille 2007 ; 219 : 127-38.

Pierre J. Vers des relations durables entre insectes auxiliaires, cultures et protection intégrée. $\mathrm{OCL}$ $2009 ; 16: 164-8$

Pierre J, Emeillat R. Les variétés de colza actuelles sont-elles peu nectarifères pour les abeilles? Bull Tech Apic 2009 ; 36 : 17-24.

Pierre J, Marsault D, Genecque E, Renard M, Champolivier J, Pham-Delègue MH. Effects of herbicide tolerant transgenic oilseed rape genotypes on honeybees and other pollinating insects under field conditions. Entomol Exp Applic $2003 ; 108$ : 159-68.

Pierre J, Mesquida J, Marilleau R, Pham-Delègue MH, Renard M. Nectar secretion in winter oilseed rape, Brassica napus - quantitative and qualitative variability among 71 genotypes. Plant Breeding $1999 ; 118$ : 471-6.

Pierre J, Mesquida J, Renard M, Vallée P. Etude de dispositifs en bandes alternées pour la production de semences de colzas hybides demi-nains en présence d'abeilles. OCL $1999 ; 6: 343-8$.

Pierre J, Pham-Delègue $\mathrm{MH}$. Comment étudier l'impact de colzas transgéniques sur les abeilles. OCL $2000 ; 7: 341-4$.

Pierre J, Pham-Delègue MH. Utilisation et protection des insectes pollinisateurs. In: Boissy A, PhamDelègue $\mathrm{M}-\mathrm{H}$, Baudoin $\mathrm{C}$, eds. Ethologie appliquée. Paris (France) : Quae, 2009.

Pierre J, Picault $\mathrm{H}$, Renard M. Relative contribution of insects and wind to seed prodcution of oilseed rape Brassica napus. Plant Breeding 2010 (sous presse).
Pierre J, Pierre JS, Marilleau R, Pham-Delègue $M H$, Tanguy $X$, Renard $M$. Influence of the apetalous character in rape (Brassica napus) on the foraging behaviour of honeybees (Apis mellifera). Plant Breeding 1996 ; 115 : 484-7.

Pierre J, Renard M. Incidence du caractère apétale chez le colza (Brassica napus L. var oleifera) sur le comportement de butinage de l'abeille domestique (Apis mellifera $\mathrm{L}$ ): étude préliminaire. Apidologie $1994 ; 25$ : 422-6.

Pierre J, Renard M. Pollinisation entomophile et production de semences hybrides chez le colza. France : ANAMSO ed. 1995.

Pierre J, Renard M. Relation between pollen deposition by honeybees and their pollen loading postures on rapeseed flowers. In : Proc. Symposium on Brassicas, 23-27 septembre 1997, Rennes.

Pierre J, Renard M. Does short distance isolation reduce pollen dispersal by honey bees? . In : Proc. Xth International Rapeseed congress, 26-29 September 1999, Canberra, Australia (CD Rom, 5 p).

Pierre J, Renard M. Plant development mutants: incidence on honey bees behaviour and pollination. In : Proc. Xth International Rapeseed congress, 26-29 September 1999, Canberra, Australia (CD Rom, $5 p$ ).

Pierre J, Renard M. La longévité du pollen de colza. OCL $2002 ; 9: 11$

Pierre J, Vaissière B, Vallée P, Renard M. Efficiency of airborne pollen released by honeybee foraging on pollination in oilseed rape : a wind insect-assisted pollination. Apidologie 2010 ; 41 : 109-15.

Rakow G, Woods DL. Outcrossing in rape and mustard under Saskatchewan Prairies conditions. Can J Plant Sci 1987 ; 67 : 147-51.

Renard M, Mesquida J. Pollinisation entomophile du colza mâle-stérile en production de semences hybrides F1 dans différentes régions de France. In : Vlème Congrès International sur le Colza, Paris, 18-19 mai 1983.

Renard M, Mesquida J. Importance relative des sécrétions nectarifères sur le comportement de butinage des abeilles et la pollinisation du colza mâle-stérile en production de semences. Bull Tech Apic 1987 ; 14 : 93-8.

Renard M, Mesquida J, Pelan-Delourme R, Pelletier G, Morice J. Pollinisation des cybrides mâle-stériles dans un système de cultures mixte de colza. In : Proc. Variabilité génétique cytoplasmique et stérilité mâlecytoplasmique, Sainte Sabine (France) 22-23 avril 1987. Colloque Inra 45 ed.

Renard M, Tanguy X. Obtention de mutants cléistogames de crucifères. Brevet FR $1997 ; 97: 15768$

Sabbahi R, De Oliveira D, Marceau J. Influence of honey bee (Hymenoptera: Apidae) density on the production of canola (Cruciferae: Brassicacae). J Econ Entomol 2005 ; 98 : 367-72. 